\title{
Bcl-2 overexpression contributes to laryngeal carcinoma cell survival by forming a complex with Hsp90ß
}

\author{
SAI LI $^{12^{*}}$, JINCHENG LI $^{3 *}$, TIAN HU ${ }^{1}$, CHUHONG ZHANG $^{1}$, XIU LV $^{1}$, SHA HE $^{1}$, HANXING YAN $^{1}$, \\ YIXI TAN $^{1}$, MEILING WEN $^{1}$, MINGSHENG LEI ${ }^{4}$ and JIANHONG ZUO ${ }^{1,2}$ \\ ${ }^{1}$ Oncology Department, The Affiliated Nanhua Hospital, University of South China, Hengyang, \\ Hunan 421001; ${ }^{2}$ School of Medicine, University of South China, Hengyang, Hunan 421001; ${ }^{3}$ Medical School, \\ Shaoyang University, Shaoyang, Hunan 422000; ${ }^{4}$ Department of Respiratory and Critical Care Medicine, \\ The People's Hospital of Zhangjiajie City, Zhangjiajie, Hunan 427000, P.R. China
}

Received May 10, 2016; Accepted July 19, 2016

DOI: 10.3892/or.2016.5295

\begin{abstract}
Laryngeal carcinoma (LC) is one of the most common malignant tumors of all head and neck squamous cell carcinomas (HNSCCs). However, the molecular mechanism and genetic basis of the development of LC have not been fully elucidated. To explore the possible mechanism, targeted proteomic analysis was performed on Bcl-2-associated proteins from LC cells. According to our results, 35 proteins associated with Bcl-2 were identified and Hsp90 $\beta$ was confirmed by co-immunoprecipitation and western blot analysis. Protein-protein interaction (PPI) analysis indicated that Bcl-2-Hsp90 $\beta$ interactions may be involved in the anti-apoptotic progression of LC. Further results revealed that disruption of the Bcl-2-Hsp90 $\beta$ interaction inhibited the anti-apoptotic ability of Bcl-2 and decreased the caspase activation in LC, which has broad implications for the better understanding of tumor formation, tumor cell survival and development of metastasis due to Bcl-2. Collectively, we report the mechanism by which Bcl-2 functions in LC as an anti-apoptotic factor in relation to its association with proteins and potentially identify a Bcl-2/Hsp90 $\beta$ axis as a novel target for LC therapy.
\end{abstract}

\section{Introduction}

Head and neck squamous cell carcinoma (HNSCC) is a heterogeneous disease composed of hypopharyngeal, oropharyngeal,

Correspondence to: Professor Mingsheng Lei, Department of Respiratory and Critical Care Medicine, The People's Hospital of Zhangjiajie City, 192 Gu Yong Road, Zhangjiajie, Hunan 427000, P.R. China

E-mail: 2846931246@qq.com

Professor Jianhong Zuo, School of Medicine, University of South China, 28 Changsheng Road, Hengyang, Hunan 421001, P.R. China E-mail: 632138414@qq.com

*Contributed equally

Key words: laryngocarcinoma cancer, Bcl-2, Hsp90 $\beta$ oral, and laryngeal squamous cell carcinoma. Laryngeal carcinoma (LC) is one of the most common malignant tumors among HNSCCs. As an etiologically multifactorial disease, carcinogenesis of laryngeal carcinoma may result from genetic and environmental factors (1). Previous study on the origin of LC suggests that genetic alterations in tumor-suppressor genes and proto-oncogenes in multiple cellular pathways may be important in multistage LC carcinogenesis. However, the molecular mechanism and genetic basis of the development of LC have not fully been elucidated.

Overexpression of Bcl-2 in primary tumors is associated with tumor cell differentiation, tumor metastasis, recurrence, and poor prognosis in patients (2) and appears to suggest apoptosis resistance in many types of cancer (3), including HNSCC (4-6). Overexpression of Bcl-2 is also related to chemotherapy resistance (4). However, molecular targeting of Bcl-2 with small-molecule inhibitors or short peptides was found to promote apoptosis and chemosensitivity in HNSCC cells $(7,8)$. These studies suggest that Bcl-2 plays a crucial role in the development and progression of cancer.

In the present study, we report the mechanism by which Bcl-2 functions in LC as an anti-apoptotic factor in relation to its association with proteins by proteomes. Our data provide novel evidence that Hsp90 $\beta$ is associated with $\mathrm{Bcl}-2$ and that this interaction facilitates optimal Bcl-2 anti-apoptotic function. Further results showed that disruption of Bcl-2-Hsp90 $\beta$ interaction inhibited the anti-apoptotic ability of Bcl-2 and decreased the caspase activation in LC, which will have broad implications for the better understanding of tumor formation, tumor cell survival, development of metastasis due to Bcl-2 and potentially identify a Bcl-2/Hsp90 $\beta$ axis as a novel target for LC therapy.

\section{Materials and methods}

Reagents. Mouse monoclonal anti-Bcl-2, anti-GAPDH, and anti-Hsp90 $\beta$ antibodies were purchased from Santa Cruz Biotechnology (Santa Cruz, CA, USA). Anti-mouse antibody was purchased from Abcam, Inc. (Cambridge, MA, USA). Mercaptoethanol, iodoacetamide, and $\mathrm{HCl}$ were purchased from Sigma-Aldrich (St. Louis, MO, USA). 
Bromophenol blue, bis, TEMED, Commassie Brilliant Blue G-250, molecular weight marker, Tris-base, SDS, glycine, secondary antibodies conjugated with horseradish peroxidase, and the enhanced chemiluminescence (ECL) system were obtained from Amersham Biosciences (Stockholm, Sweden). Sequencing-grade modified trypsin was purchased from Promega Corp. (Madison, WI, USA). PVDF membranes and ZipTip $\mathrm{C}^{18}$ columns were obtained from Millipore Corp. (Boston, MA, USA).

Cell and cell culture. The HNSCC cell line SCC10A was derived from the primary lesion of a larynx carcinoma, and has been extensively characterized for its in vitro and in vivo phenotypes (9). Cells were normally maintained at low passage in Dulbecco's modified Eagle's medium (DMEM) supplemented with 10\% fetal bovine serum (FBS) (both from Invitrogen), $100 \mathrm{U} / \mathrm{ml}$ penicillin and $100 \mu \mathrm{g} / \mathrm{ml}$ streptomycin.

Co-immunoprecipitation. LC SCC10A cells were lysed at $4^{\circ} \mathrm{C}$ for $30 \mathrm{~min}$ in a lysis buffer $[50 \mathrm{mmol} / 1$ Tris $(\mathrm{pH} 7.5)$, $500 \mathrm{mmol} / 1 \mathrm{NaCl}, 1 \%$ Triton $\mathrm{X}-100,0.5 \%$ sodium deoxycholate, $0.1 \%$ SDS, $10 \mathrm{mmol} / 1 \mathrm{MgCl}_{2}$, and complete protease inhibitor mixture (Roche Molecular Biochemicals, Mannheim, Germany)]. The lysates were centrifuged at $11,000 \mathrm{rpm}$ for $15 \mathrm{~min}$ at $4^{\circ} \mathrm{C}$. Protein concentrations were measured with the bicinchoninic acid protein assay kit (Pierce, Rockford, IL, USA). The clarified supernatants were collected and used immediately for co-immunoprecipitation. Approximately $500 \mathrm{mg}$ of total protein was first precleared with control (non-immune) serum, bound to $100 \mu \mathrm{l}$ protein G-Sepharose (Amersham Biosciences). The clarified supernatants were then incubated with the anti-Bcl-2 antibody $(10 \mu \mathrm{g})$ for $6 \mathrm{~h}$. Protein G-Sepharose $(100 \mu \mathrm{l})$ was added and the mixture was incubated overnight at $4^{\circ} \mathrm{C}$. Samples were centrifuged for $30 \mathrm{sec}$ and washed three times with lysis buffer, and run on a SDS-PAGE. Subsequently, SDS-PAGE electrophoresis was performed and proteins in the gels were detected by Coomassie blue R-250 staining, followed by in-gel trypsin digestion and MS analysis as previously described by us (10). For western blot analysis, proteins in the gels were transferred to nitrocellulose membranes (Millipore Corp.). Then the membranes were incubated with an anti-Bcl- 2 or an anti-Hsp90 $\beta$ antibody. The Bcl-2 antibody was then replaced by the non-immune IgY antibody (GenWay Biotech, Inc., San Diego, CA, USA) which was used as a negative control.

MS and database analysis. ESI-Q-TOF-MS analysis of proteins was performed as described by Cheng et al and Huang et al, respectively $(10,11)$.

Western blot analysis. The immunoprecipitated complexes or cell lysates were separated by $10 \%$ SDS-PAGE, and transferred to nitrocellulose membranes (Millipore Corp.). Blots were blocked with $5 \%$ non-fat dry milk for $30 \mathrm{~min}$ at room temperature and washed three times with phosphate-buffered saline (PBS) buffer. Then they were incubated with primary anti-Bcl-2, anti-Hsp90 $\beta$, or anti-GAPDH antibodies overnight at $4^{\circ} \mathrm{C}$, followed by incubation with a horseradish peroxidase-conjugated secondary antibody for $1 \mathrm{~h}$ at room temperature. The signal was visualized with ECL detection reagent. GAPDH was detected simultaneously using mouse anti-GAPDH antibody as a loading control.

Bioinformatic analysis. Molecule function classification and cluster analysis were performed through the GO and Cluster program DAVID (http://david.abcc.ncifcrf.gov/). The default parameters of classification in terms of stringency in the DAVID Cluster program were medium. Protein-protein interaction (PPI) analysis was performed using VisANT software (version 3.15) (http://visant.bu.edu/) (12).

RNA interference analysis. For RNA interference analysis, the cells were transfected with Hsp $90 \beta$ siRNA or control siRNA (Dharmacon, Inc.) using the Lipofectamine 2000 reagent (Invitrogen) according to the siRNA transfection protocol provided by the manufacturer. Briefly, the day before transfection, SCC10A were plated into 6-well plates at the density of $10^{5}$ cells $/ \mathrm{ml}$ in DMEM containing $10 \%$ FBS. When the cells reached $60-80 \%$ confluence, they were transfected with $10 \mathrm{nmol} / 1$ of Hsp $90 \beta$ siRNA or control siRNA after a preincubation for 20 min with siRNA transfection reagent in siRNA transfection medium. Four hours after the beginning of the transfection, the medium was replaced with DMEM containing 10\% FBS and the cells continued to culture for an additional $44 \mathrm{~h}$. At the end of the transfection, the Hsp90 $\beta$ expression level in the cells was determined by western blot analysis.

Caspase activity assay. The caspase-3/7 fluorometric assay kit (Promega Corp.) was used to measure caspase-3/7 activity following the manufacturer's instructions.

Immunohistochemistry. Immunohistochemical analysis of Bcl-2 and Hsp90 $\beta$ was carried out with formalin-fixed and paraffin-embedded tissue sections using the standard immunohistochemical technique according to a report by Zuo et al (13).

Statistical analysis. A Student's t-test was used for the statistical analysis, with $\mathrm{P}<0.05$ considered as a significant difference.

\section{Results}

Bcl-2-associated proteins are identified by co-immunoprecipitation and $M S$. To isolate proteins that interact with Bcl-2, we performed a proteomic analysis of the Bcl-2 complexes using targeted proteomics (co-immunoprecipitation coupled with MS). The complex was eluted, separated on an SDS-PAGE (Fig. 1A), and subjected to in-gel trypsin digestion. The tryptic digests were analyzed through ESI-Q-TOF-MS. To control for non-specific immune complexes, the Bcl-2 antibody was replaced by a non-immune IgY antibody which was used as a negative control. Thirty-five proteins were identified in the Bcl-2 complex after removing the proteins that were not found in the replicate experiments, and subtracting the common proteins that were found in the negative control (Table I).

All 35 protein bands were excised from the stained gels, in situ digested with trypsin and analyzed by MALDI-TOF MS. A total of 35 differential protein bands were identified. 
A

B

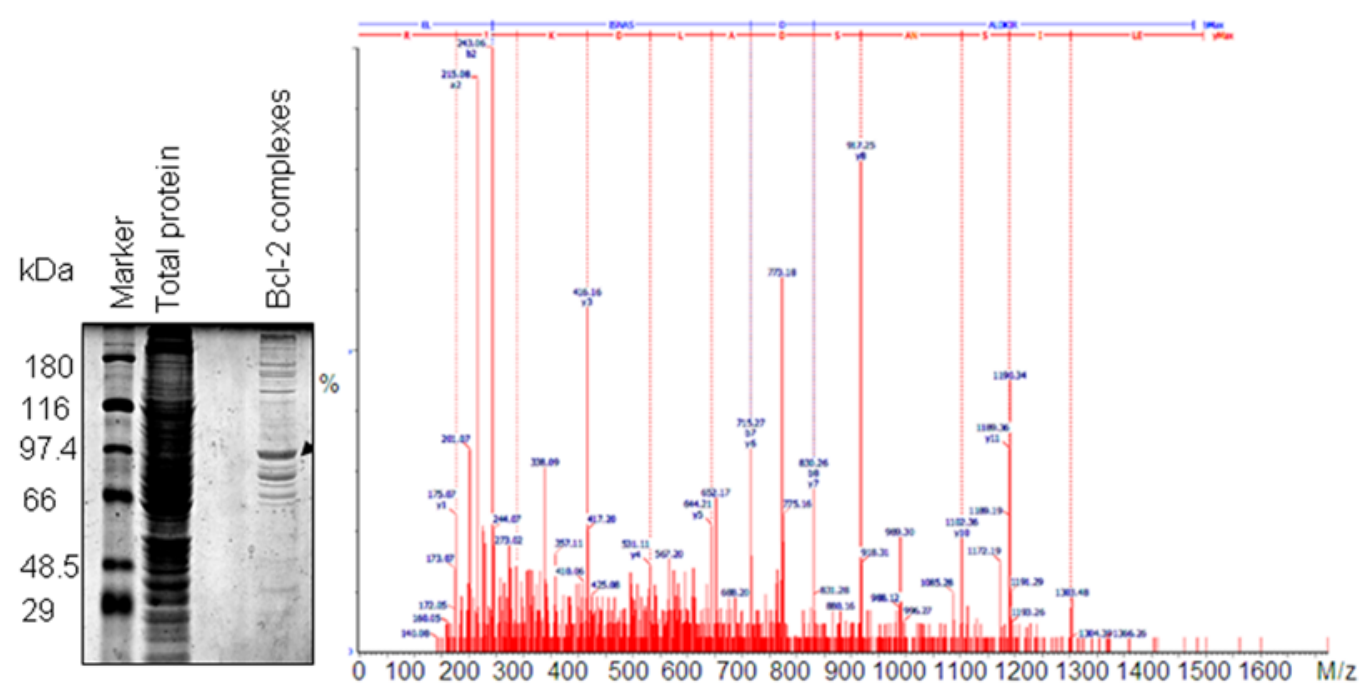

C

Mass: 83527 Score: 240

Heat shock protein HSP 90-beta OS=Equus Queries matched: 5

caballus $\mathrm{GN}=\mathrm{HSP} 90 \mathrm{AB} 1 \mathrm{PE}=2 \mathrm{SV}=3$
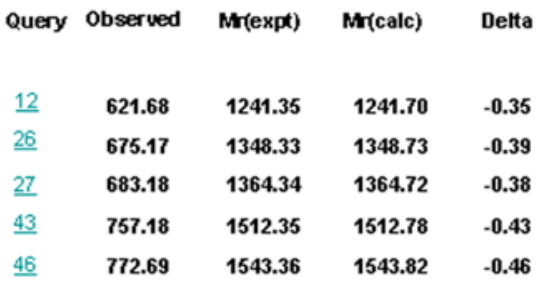

Miss Score

Expect

Rank Peptide

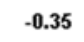

$-0.39$

0 44

$\mathbf{0 . 0 0 5}$

0.011

1 ADLIHHLGTIAK

$-0.38$

$\begin{array}{rrr}0 & 41 & 0.011 \\ 0 & (27) & 0.27\end{array}$

1 TLTLVDTGIGMTK

1 TLTLVDTGIGMTK + Oxidation (M)

772.69

1543.82

$-0.46$

$\begin{array}{lll}0 & 76 & 2.9 \mathrm{e}-006\end{array}$

1 GVUDSEDLPLHISR

1 ELISHASDALDKIR

\section{Probability Based Mowse Score}

Ion score is $-10^{*} \log (P)$, where $P$ is the probability that the observed match is a random event. Individual ion scores $>33$ indicate identity or extensive homology $(p<0.05)$.

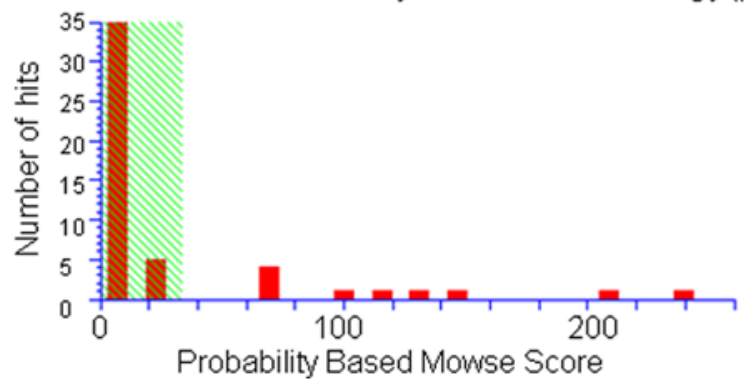

Figure 1. MALDI-TOF MS analysis of Hsp90ß. (A) Identification of Bcl-2-associated proteins by co-immunoprecipitation and MS. SDS-PAGE electrophoresis of Bcl-2 co-immunoprecipitated complex. Proteins in the gels were detected by Coomassie blue R-250 staining, followed by in-gel trypsin digestion and mass spectrometric analysis. (B) The MALDI-TOF MS mass spectrum identified as Hsp90 $\beta$ according to the matched peaks is shown. (C) The protein sequence of Hsp90 $\beta$ is shown, and the matched peptides are indicated in red. (D) Database query result and score of protein Hsp90 $\beta$.

The MALDI-TOF mass spectrometry map and database query result of the representative bands of $\mathrm{Hsp} 90 \beta$ are presented in Fig. $1 \mathrm{~B}$ and $\mathrm{C}$. The total of the monoisotopic peaks was input into the Mascot search engine to search the Swiss-Prot database, and the query result showed that the protein bands belonged to Hsp90 $\beta$ (Fig. 1C and D). The annotation of all the identified proteins is summarized in Table I.

Validation of the interactome of Bcl-2. To confirm the MS analysis results, we selected the protein of interest, Hsp90 $\beta$, and detected its binding to Bcl-2 by co-immunoprecipitation and western blot analysis based on the availability and quality of the available antibodies. As shown in Fig. 2A, Hsp90 $\beta$ was detected in the Bcl-2 immune complex but not in the control. Concurrently, Bcl-2 was detected in the Hsp90 $\beta$ immune complex but not in the control (Fig. 2B). These data showed that the MS analysis results are reliable for bioinformatics and PPI analysis.

PPI analysis. In order to understand the interaction among the Bcl-2-associated proteins, PPI analysis was performed using VisANT software (http://visant.bu.edu/), a data integrating 
Table I. Bcl-2 interacting proteins.

\begin{tabular}{|c|c|c|c|c|}
\hline $\begin{array}{l}\text { Protein accession } \\
\text { nos. }\end{array}$ & $\begin{array}{l}\text { Protein molecular } \\
\text { weight }(\mathrm{Da})\end{array}$ & $\begin{array}{c}\text { No. of } \\
\text { unique peptides }\end{array}$ & Score & $\begin{array}{c}\text { Percentage sequence } \\
\text { coverage }(\%)\end{array}$ \\
\hline 4F2_HUMAN & $67,978.40$ & 2 & 101 & 4.13 \\
\hline \multicolumn{5}{|l|}{ ACTN1_HUMAN, } \\
\hline ACTN4_HUMAN & $103,043.00$ & 2 & 120 & 2.69 \\
\hline ALBU_HUMAN & $69,348.90$ & 2 & & 2.46 \\
\hline COF1_HUMAN & $18,485.10$ & 2 & 62 & 15.10 \\
\hline DDX1_HUMAN & $82,415.10$ & 2 & 127 & 3.51 \\
\hline DDX3X_HUMAN & $73,227.70$ & 2 & 83 & 3.93 \\
\hline DDX5_HUMAN & $69,131.70$ & 3 & 137 & 6.51 \\
\hline EIF3L_HUMAN & $66,711.30$ & 2 & & 5.14 \\
\hline GRP78_HUMAN & $72,316.70$ & 3 & 115 & 6.57 \\
\hline GTF2I_HUMAN & $112,399.90$ & 2 & 41 & 2.71 \\
\hline GTF2I_HUMAN & $112,399.90$ & 3 & 149 & 4.01 \\
\hline HS90ß_HUMAN & $83,249.30$ & 4 & 240 & 7.32 \\
\hline HSP7C_HUMAN & $70,881.80$ & 5 & 229 & 11.50 \\
\hline K2C1_HUMAN & $66,022.30$ & 3 & 133 & 6.37 \\
\hline K2C5_HUMAN & $62,361.60$ & 1 & 46 & 2.37 \\
\hline K2C5_HUMAN & $62,361.60$ & 3 & 126 & 6.44 \\
\hline MYH9_HUMAN & $226,519.50$ & 2 & 85 & 1.12 \\
\hline PI51A_HUMAN & $62,617.40$ & 3 & 137 & 6.94 \\
\hline PKP3_HUMAN & $87,066.70$ & 2 & 65 & 3.76 \\
\hline PLEC1_HUMAN & $531,765.90$ & 8 & 361 & 1.79 \\
\hline PSPC1_HUMAN & $58,726.50$ & 5 & 236 & 11.10 \\
\hline RL11_HUMAN & $20,235.20$ & 1 & 55 & 7.87 \\
\hline RL22_HUMAN & $14,769.30$ & 1 & 46 & 8.59 \\
\hline RS16_HUMAN & $16,427.90$ & 2 & 67 & 15.10 \\
\hline RS23_HUMAN & $15,789.70$ & 1 & 90 & 7.69 \\
\hline SFPQ_HUMAN & $76,131.50$ & 2 & 75 & 2.97 \\
\hline TBB5_HUMAN & $49,652.60$ & 4 & 134 & 11.70 \\
\hline
\end{tabular}

visual framework for biological networks and modules. Entrez-gene IDs of $35 \mathrm{Bcl}-2$-associated proteins were input into the VisANT software and a complex net was obtained. The PPIs are presented in Fig. 3A and it was revealed that Bcl-2 could interact with Hsp90 $\beta$. In order to detect the relationship between Hsp90 $\beta$ and susceptibility to apoptosis in LC cells, we chose the Bcl-2/Hsp90 $\beta$ interaction for further study.

Hsp90 $\beta$ expression is necessary for Bcl-2 anti-apoptosis. In order to detect whether Hsp90 $\beta$ is important to the anti-apoptotic function of Bcl-2, we applied geldanamycin (GA), as a specific inhibitor of Hsp90 $\beta$, to study the chaperone function of Hsp90 $\beta$ in its association with Bcl-2. LC cells were treated with various concentrations of GA for $24 \mathrm{~h}$. As expected, the data showed that the association of Hsp90 $\beta$ with Bcl-2 was effectively blocked in a dose-dependent manner by GA in the Bcl-2 immunoprecipitation (Fig. 3B). Moreover, caspase-3/7 activities in the cells treated with GA showed a dose-dependent increase (Fig. 3C), which suggested that the inhibition of the chaperone function of Hsp90 $\beta$ in its association with Bcl-2 decreased the ability of the anti-apoptotic efffect of Bcl-2.
A

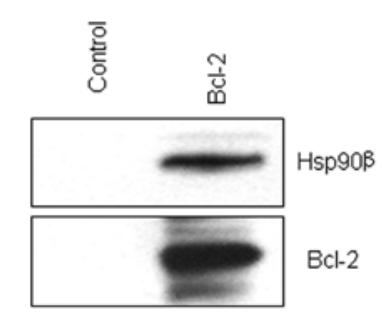

B

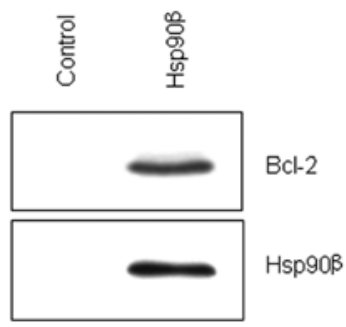

Figure 2. Analysis of the Bcl-2 interactome in the SCC10A laryngeal carcinoma (LC) cells. (A) Conformation of Bcl-2-associated proteins by co-immunoprecipitation and western blot analysis. Bcl-2 and Hsp90 $\beta$ were detected in the Bcl-2 coimmunoprecipitated complex but not in the nonimmune IgY control. (B) Conformation of Hsp90 $\beta$-associated proteins by co-immunoprecipitation and western blot analysis. Hsp90 $\beta$ and Bcl-2 were detected in the Hsp90 $\beta$ co-immunoprecipitated complex but not in the non-immune IgY control.

Knockdown of Hsp90 $\beta$ decreases the ability of anti-apoptosis by Bcl-2. To further study the function of the Hsp90 $\beta$-Bcl-2 association, specific siRNA of Hsp90 $\beta$ was carried out to knock down the expression of Hsp90 $\beta$. The results revealed that 


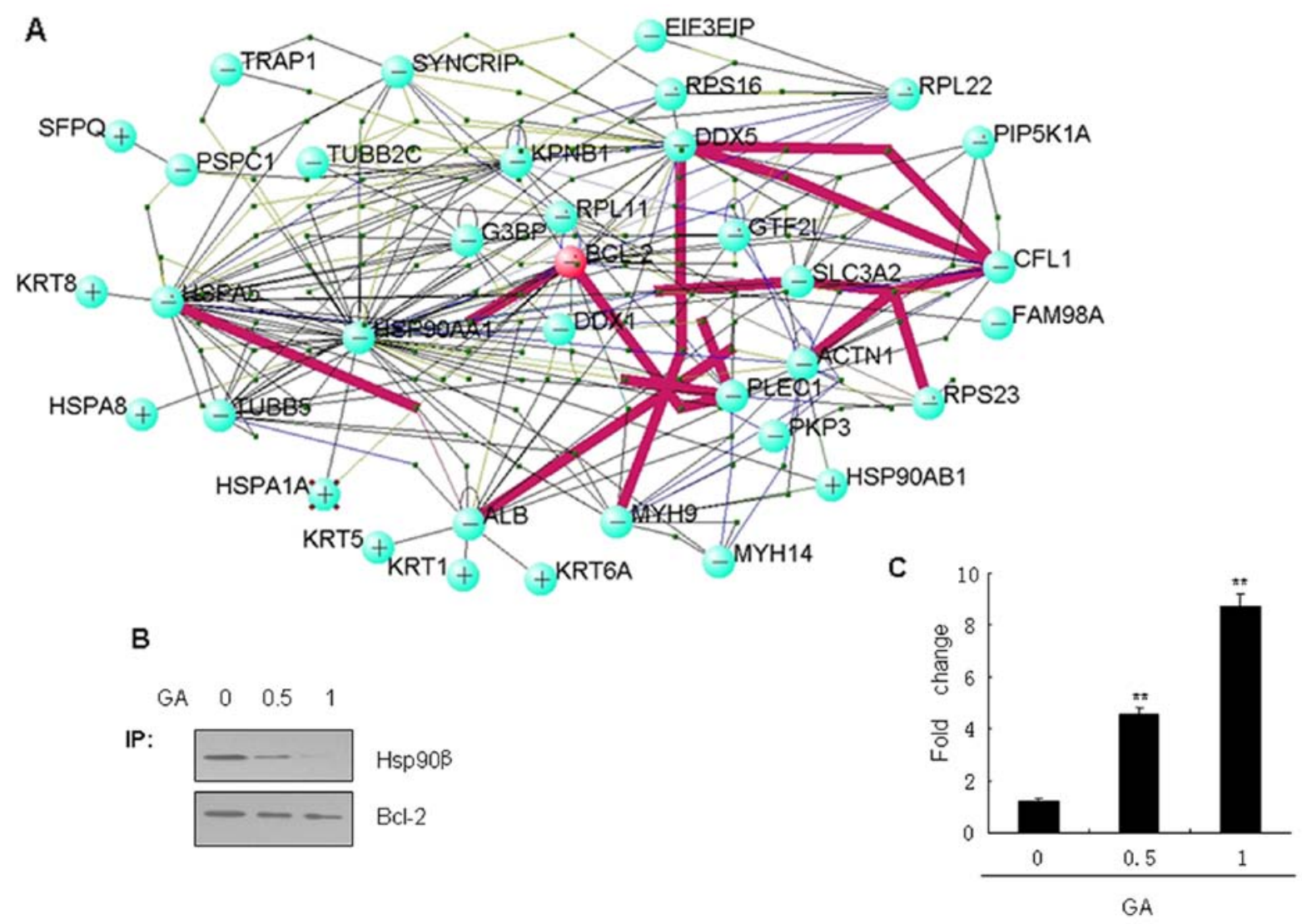

Figure 3. Inhibition of Hsp90 $\beta$ increases laryngeal carcinoma (LC) cell apoptosis. (A) Analysis of protein-protein interaction using VisANT software (http://visant.bu.edu/). General arrangement of all the interactions of Bcl-2-associated proteins is presented. Spheres marked with alias or entrez-gene IDs are the identified Bcl-2-associated proteins. Proteins marked by a large red sphere and interactions marked by thick lines represent the foregone interactions of the $\mathrm{Bcl}-2$-associated proteins. +, there are interactions with the node proteins not displayed in the image. -, all known interactions with the node proteins are displayed in the image. [For interpretation of the references to color in this figure legend, the reader is referred to the web version of the article (http://visant. bu.edu/)]. (B) SCC10A cells were treated with geldanamycin (GA). After $24 \mathrm{~h}$, whole-cell lysates were prepared and Hsp90 $\beta$ expression was analyzed by cell lysates which were also subjected to immunoprecipitation (IP) with anti-Bcl-2 and detected by immunoblotting with an antibody to Hsp90 3 . (C) Inhibition of Hsp90 $\beta$ increased caspase-3/7 activity in the SCC10A cells. ${ }^{* * *} \mathrm{P}<0.001$.

A

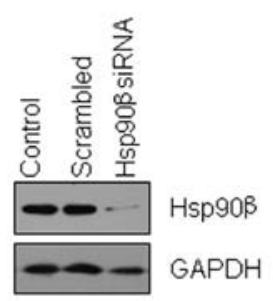

C

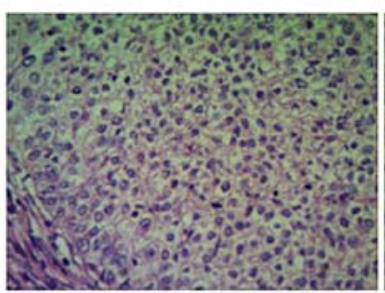

H\&E
B

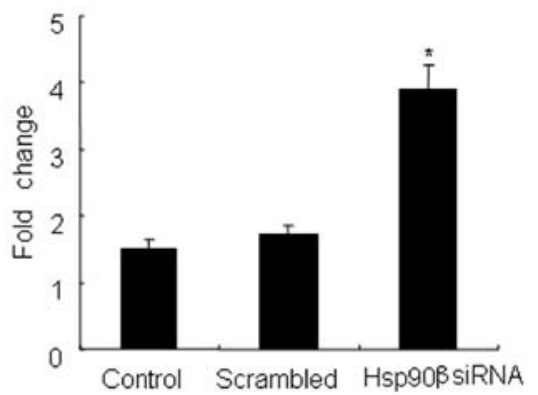

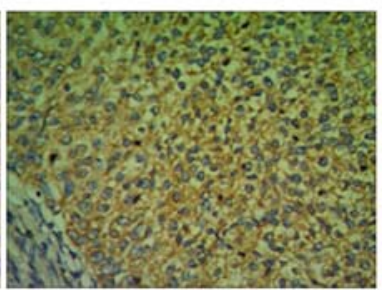

$\mathrm{Bcl}-2$

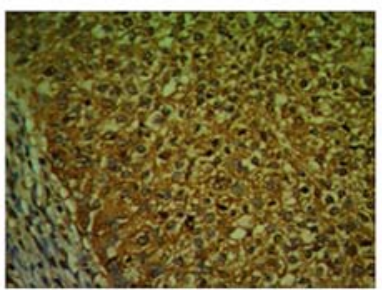

HSP9OB

Figure 4. Knockdown of Hsp90 $\beta$ increases laryngeal carcinoma (LC) cell apoptosis. (A) SCC10A cells were left untreated or transfected with scrambled or Hsp90 $\beta$ siRNA. After $48 \mathrm{~h}$, whole-cell lysates were prepared and the expression of Hsp90 $\beta$ was analyzed by immunoblotting. GAPDH was used as a loading control. (B) Knockdown of Hsp90ß increased caspase-3/7 activity in the SCC10A cells. ${ }^{*} \mathrm{P}<0.05$. (C) Immunohistochemical analysis of the expression of Bcl-2 and $\mathrm{Hsp} 90 \beta$ proteins in the LC tissues. 
Table II. Correlation between Bcl-2 and Hsp90 $\beta$ expression in LC.

\begin{tabular}{lrr}
\hline & \multicolumn{2}{c}{ Hsp90ß } \\
\cline { 2 - 3 } & + & - \\
\hline Bcl-2 & & \\
+ & 38 & 2 \\
- & 6 & 11 \\
\hline
\end{tabular}

LC, laryngeal carcinoma.

the protein level of Hsp90 $\beta$ was significantly decreased $48 \mathrm{~h}$ after the treatment with the specific Hsp90 $\beta$ RNAi. However, the change in expression of Hsp90 $\beta$ was not detected in the untreated cells or the cells treated with scrambled siRNA as determined by western blot analysis (Fig. 4A). Concurrently, it is of note that the knockdown of Hsp90 $\beta$ expression led to a significant increase in the activity of caspase- $3 / 7$ in the SCC10A cells compared to the control cells (Fig. 4B).

Correlation between Bcl-2 and Hsp90 $\beta$ expression and clinicopathological factors in $L C$. In order to further verify whether the Bcl-2 target we identified is also associated with Hsp90 $\beta$ in vivo, we examined the expression levels of the Bcl-2 and Hsp90ß proteins, which are both actually highly expressed in LC (Fig. 4C). By Spearman correlation analysis it was concluded that there was a positive correlation between the expression of $\mathrm{Bcl}-2$ and $\mathrm{Hsp} 90 \beta$ in laryngeal squamous cell carcinoma $(\mathrm{P}<0.05)$ (Table II). The expression of $\mathrm{Bcl}-2$ and $\mathrm{Hsp} 90 \mathrm{\beta}$ in LC tissues was not associated with the age and gender of the patients $(\mathrm{P}>0.05)$ whereas it was correlated with tumor differentiation, clinical stage and lymph node metastasis $(\mathrm{P}<0.05)$ (Table III).

\section{Discussion}

Human Bcl-2 is located near the junction of chromosomes 18 and $14(\mathrm{t} 14 ; 18)$ and has been discovered in the tumor cells of follicular lymphoma patients. The chromosome translocation results in misregulation of the normal Bcl-2 expression pattern, which leads to abnormal cell growth and certainly contributes to the development of certain types of tumors $(14,15)$. Bcl-2 overexpression occurs in a wide range of human cancers (16) and causes resistance to apoptosis, autophagic-associated cell death and treatment (16-19). Elevated expression of Bcl-2 in some tumors is often associated with enhanced invasion and metastasis (20-22), shorter survival time and generally poorer clinical outcomes (23). Thus, Bcl-2 plays an important role in the orientation and development of tumors. Bcl-2 is one of the key regulators of apoptosis because it endows a survival advantage on cells by protecting cells from apoptotic death (17). However, this unknown mechanism needs to be elucidated, especially in LC. In the present study, we investigated in detail, the molecular pathways by which $\mathrm{Bcl}-2$ overexpression contributes to LC cell survival by forming a complex with Hsp90ß (Fig. 5).
Table III. Correlation between Bcl-2 and Hsp90 $\beta$ expression and clinicopathologic factors in LC.

\begin{tabular}{|c|c|c|c|c|c|c|}
\hline \multirow[b]{2}{*}{ Parameter } & \multicolumn{2}{|c|}{ Bcl-2 } & \multirow[b]{2}{*}{ P-value } & \multicolumn{2}{|c|}{ Hsp90 $\beta$} & \multirow[b]{2}{*}{ P-value } \\
\hline & - & + & & - & + & \\
\hline Overall & 17 & 40 & & 13 & 44 & \\
\hline Age (years) & & & 0.340 & & & 0.381 \\
\hline$>60$ & 7 & 22 & & 8 & 21 & \\
\hline$\leq 60$ & 10 & 18 & & 5 & 23 & \\
\hline Gender & & & 0.279 & & & 0.240 \\
\hline Male & 11 & 36 & & 8 & 39 & \\
\hline Female & 6 & 4 & & 5 & 5 & \\
\hline $\begin{array}{l}\text { Tumor } \\
\text { differentiation }\end{array}$ & & & 0.023 & & & 0.006 \\
\hline Well/moderate & 14 & 20 & & 12 & 22 & \\
\hline Poor & 3 & 20 & & 1 & 22 & \\
\hline $\begin{array}{l}\text { Lymph node } \\
\text { metastasis }\end{array}$ & & & 0.014 & & & 0.010 \\
\hline Yes & 5 & 26 & & 3 & 28 & \\
\hline No & 12 & 14 & & 10 & 16 & \\
\hline TNM stage & & & 0.025 & & & 0.002 \\
\hline I-II & 7 & 29 & & 13 & 23 & \\
\hline III-IV & 10 & 11 & & 0 & 21 & \\
\hline
\end{tabular}

LC, laryngeal carcinoma.

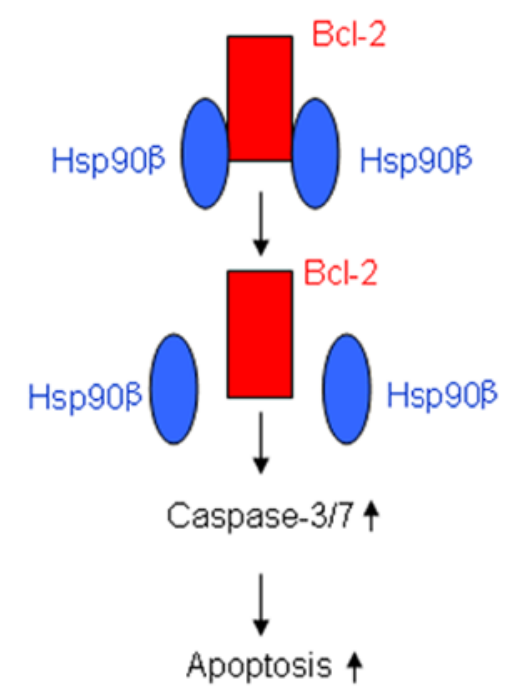

Figure 5. Model of Bcl-2 function in laryngeal carcinoma cells.

VisANT extends the application of the GO database, in network visualization, analysis and inference as an integrative software platform used for the visualization, mining, analysis and modeling of biological networks $(12,24)$. VisANT supports biological network mining and analysis, meaning that it is able to find previously unidentified networks, annotate them, and display their hierarchical organization (25). 
VisANT allows the different types of networks to analyze the correlations between disease, therapy, genes and drugs systematically (26). VisANT's ability to use nodes to model more complex entities such as protein complexes or pathways allows for more informative visualizations. VisANT also implements algorithms for analyzing node degrees, clusters, path lengths, network motifs and network randomizations (27). In this study, PPI was performed using VisANT software to discover how $\mathrm{Bcl}-2$ interacts with its associated proteins. The results indicated that $\mathrm{Bcl}-2$ interacts with $\mathrm{Hsp} 90 \beta$, which regulates cell apoptosis.

Heat shock proteins represent a diverse group of chaperones that play a critical role in the protection of cells against numerous environmental stresses (27). Heat shock proteins also carry out the functions of the folding of synthesized proteins, and play a role in the maturation and activity of many proteins. Many Hsp90 clients such as Raf, Bcr-Abl, and C-kit are oncoproteins that are either mutated or overexpressed in cancer cells, which in turn lead to the disregulation of cell growth and proliferation (28). Hsp90 can regulate cell motility, inflammation, angiogenesis, matrix remodeling, tumor progression and metastasis (27). Hsp90 $\beta$ is one of the isoforms of Hsp90, which plays an important role in forming a multiprotein complex with ATPase activity. It is also involved in the folding, activation and assembly of several proteins, such as the tumor-suppressor protein p53, the NOS family members, Akt/protein kinase B and Raf-1 $(29,30)$, which result in signal transduction and transcriptional regulation. Hsp90 $\beta$ is a calcium-binding protein and a novel regulatory factor of MMP-13 expression in osteoarthritic chondrocytes (31). As many client proteins of Hsp90 control cell survival, proliferation, and apoptosis, Hsp90 is closely associated with human health, especially tumors. Concurrently, the expression of Hsp90 is 2- to 10-fold higher in tumor cells than in normal cells (32). Therefore, Hsp90 has emerged as an important target in cancers such as non-small cell lung cancer, melanoma and breast cancer (33) in recent years.

In the present study, GA, a benzoquinone ansamycin antibiotic, has been used as an inhibitor of Hsp90 $\beta$ since it specifically binds to the ATP/ADP pocket binding site in the $\mathrm{N}$-domain of Hsp90 $\beta$, which leads to disruption of the Hsp90 $\beta$ interaction with certain proteins. Thus, the GA inhibitor was applied to test the chaperone function of Hsp90 in its association with $\mathrm{Bcl}-2$. Co-immunoprecipitation experiments showed that Hsp90 $\beta$ binding to Bcl-2 was abrogated by GA, which suggests that Hsp90 $\beta$ may fail to bind to Bcl-2. We also found that decreased Hsp90 $\beta$ binding to Bcl-2 by GA led to these cells being less resistant to apoptosis than the control cells. As predicted, GA induced cell apoptosis to a greater degree when compared with the control. Notably, the data suggest that GA has a potent antitumor effect and may result in its use in clinical trials in LC in the future.

In this study, we immunohistochemically determined Bcl-2 and Hsp90 $\beta$ expression in 57 patients with LC, and found a 70.18 and $77.20 \%$ positive frequency, respectively, similar to that reported previously. Moreover, we showed that Bcl-2 and Hsp90 $\beta$ were highly expressed in tumors, and positively associated with tumor differentiation, clinical stage and lymph node metastasis. Finally we demonstrated a positive correlation between $\mathrm{Bcl}-2$ expression and $\mathrm{Hsp} 90 \beta$ expression, when the percentage of positively stained cells was determined in the LC tissues. All in all, these aforementioned findings strongly support the relationship of Bcl-2 and Hsp90 $\beta$ and their role in LC.

Collectively, in the present study we found a novel molecular mechanism of anti-apoptosis via Bcl-2/Hsp90 $\beta$ interaction in $\mathrm{LC}$, which is strong evidence for new targets for LC therapy.

\section{Acknowledgements}

This study was supported by the National Natural Science Foundation of China (no. 81272960), the Key Research Program from the Science and Technology Department of Hunan Province, China (no. 2013WK2010, 2014SK2015) and the Key Research Program from the Ministry of Human Resources and Social Security of the People's Republic of China (2016) (no. 176).

\section{References}

1. Jiang LY, Lian M, Wang H, Fang JG and Wang Q: Inhibitory effects of 5-Aza-2'-deoxycytidine and trichostatin A in combination with $p 53$-expressing adenovirus on human laryngocarcinoma cells. Chin J Cancer Res 24: 232-237, 2012.

2. Zuo J, Ishikawa T, Boutros S, Xiao Z, Humtsoe JO and Kramer RH: Bcl-2 overexpression induces a partial epithelial to mesenchymal transition and promotes squamous carcinoma cell invasion and metastasis. Mol Cancer Res 8: 170-182, 2010.

3. Kang MH and Reynolds CP: Bcl-2 inhibitors: Targeting mitochondrial apoptotic pathways in cancer therapy. Clin Cancer Res 15: 1126-1132, 2009.

4. Lu S, Yu L, Mu Y, Ma J, Tian J, Xu W and Wang H: Role and mechanism of Twist 1 in modulating the chemosensitivity of FaDu cells. Mol Med Rep 10: 53-60, 2014.

5. Wu M, Zhang H, Hu J, Weng Z, Li C, Li H, Zhao Y, Mei X, Ren F and $\mathrm{Li} \mathrm{L}$ : Isoalantolactone inhibits UM-SCC-10A cell growth via cell cycle arrest and apoptosis induction. PLoS One 8: e76000, 2013.

6. Rahman MA, Amin AR, Wang D, Koenig L, Nannapaneni S, Chen Z, Wang Z, Sica G, Deng X, Chen ZG, et al: RRM2 regulates $\mathrm{Bcl}-2$ in head and neck and lung cancers: A potential target for cancer therapy. Clin Cancer Res 19: 3416-3428, 2013.

7. Kawano K, Kantak SS, Murai M, Yao CC and Kramer RH: Integrin $\alpha 3 \beta 1$ engagement disrupts intercellular adhesion. Exp Cell Res 262: 180-196, 2001.

8. Nurmenniemi S, Sinikumpu T, Alahuhta I, Salo S, Sutinen M, Santala M, Risteli J, Nyberg P and Salo T: A novel organotypic model mimics the tumor microenvironment. Am J Pathol 175: 1281-1291, 2009.

9. Zuo JH, Zhu W, Li MY, Li XH, Yi H, Zeng GQ, Wan XX, He QY, Li JH, Qu JQ, et al: Activation of EGFR promotes squamous carcinoma SCC10A cell migration and invasion via inducing EMT-like phenotype change and MMP-9-mediated degradation of E-cadherin. J Cell Biochem 112: 2508-2517, 2011.

10. Cheng AL, Huang WG, Chen ZC, Peng F, Zhang PF, Li MY, Li F, $\mathrm{Li} \mathrm{JL}, \mathrm{Li} \mathrm{C}$, Yi H, et al: Identification of novel nasopharyngeal carcinoma biomarkers by laser capture microdissection and proteomic analysis. Clin Cancer Res 14: 435-445, 2008.

11. Huang WG, Cheng AL, Chen ZC, Peng F, Zhang PF, Li MY, Li F,

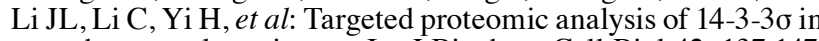
nasopharyngeal carcinoma. Int J Biochem Cell Biol 42: 137-147, 2010.

12. Hu Z, Hung JH, Wang Y, Chang YC, Huang CL, Huyck M and DeLisi C: VisANT 3.5: Multi-scale network visualization, analysis and inference based on the gene ontology. Nucleic Acids Res 37 (Web Server): W115-W121, 2009.

13. Zuo J, Wen M, Lei M, Peng X, Yang X and Liu Z: MiR-210 links hypoxia with cell proliferation regulation in human Laryngocarcinoma cancer. J Cell Biochem 116: 1039-1049, 2015.

14. Hardwick JM and Soane L: Multiple functions of BCL-2 family proteins. Cold Spring Harb Perspect Biol 5: a008722, 2013.

15. Yip KW and Reed JC: Bcl-2 family proteins and cancer. Oncogene 27: 6398-6406, 2008. 
16. Yu L and Liu S: Autophagy contributes to modulating the cytotoxicities of Bcl-2 homology domain-3 mimetics. Semin Cancer Biol 23: 553-560, 2013.

17. Marquez RT and Xu L: Bcl-2:Beclin 1 complex: multiple, mechanisms regulating autophagy/apoptosis toggle switch. Am J Cancer Res 2: 214-221, 2012.

18. Karnak D and Xu L: Chemosensitization of prostate cancer by modulating Bcl-2 family proteins. Curr Drug Targets 11: 699-707, 2010.

19. Buggins AG and Pepper CJ: The role of Bcl-2 family proteins in chronic lymphocytic leukaemia. Leuk Res 34: 837-842, 2010.

20. Mena S, Benlloch M, Ortega A, Carretero J, Obrador E, Asensi M, Petschen I, Brown BD and Estrela JM: Bcl-2 and glutathione depletion sensitizes B16 melanoma to combination therapy and eliminates metastatic disease. Clin Cancer Res 13: 2658-2666, 2007.

21. Xie L, Qin W, Li J, He X, Zhang H, Yao G, Shu H, Yao M, Wan D and Gu J: BNIPL-2 promotes the invasion and metastasis of human hepatocellular carcinoma cells. Oncol Rep 17: 605-610, 2007.

22. Planas-Silva MD, Bruggeman RD, Grenko RT and Smith JS: Overexpression of $\mathrm{c}-\mathrm{Myc}$ and $\mathrm{Bcl}-2$ during progression and distant metastasis of hormone-treated breast cancer. Exp Mol Pathol 82: 85-90, 2007.

23. Olsson Åkefeldt S, Ismail MB, Valentin H, Aricò M, Henter JI and Delprat C: Targeting BCL2 family in human myeloid dendritic cells: A challenge to cure diseases with chronic inflammations associated with bone loss. Clin Dev Immunol 2013: 701305, 2013.

24. Hu Z, Snitkin ES and DeLisi C: VisANT: An integrative framework for networks in systems biology. Brief Bioinform 9: 317-325, 2008.
25. Hu Z, Chang YC, Wang Y, Huang CL, Liu Y, Tian F, Granger B and Delisi C: VisANT 4.0: Integrative network platform to connect genes, drugs, diseases and therapies. Nucleic Acids Res 41 (Web Server): W225-W231, 2013.

26. Suderman M and Hallett M: Tools for visually exploring biological networks. Bioinformatics 23: 2651-2659, 2007.

27. Hance MW, Nolan KD and Isaacs JS: The double-edged sword: Conserved functions of extracellular hsp90 in wound healing and cancer. Cancers (Basel) 6: 1065-1097, 2014.

28. Tsaytler PA, Krijgsveld J, Goerdayal SS, Rüdiger S and Egmond MR: Novel Hsp90 partners discovered using complementary proteomic approaches. Cell Stress Chaperones 14: 629-638, 2009.

29. Calamia V, de Andrés MC, Oreiro N, Ruiz-Romero C and Blanco FJ: Hsp90b inhibition modulates nitric oxide production and nitric oxide-induced apoptosis in human chondrocytes. BMC Musculoskelet Disord 12: 237, 2011.

30. Jia W, Yu C, Rahmani M, Krystal G, Sausville EA, Dent P and Grant S: Synergistic antileukemic interactions between 17-AAG and UCN-01 involve interruption of RAF/MEK- and AKT-related pathways. Blood 102: 1824-1832, 2003.

31. Fan Z, Tardif G, Hum D, Duval N, Pelletier JP and Martel-Pelletier J: Hsp90 $\beta$ and p130cas: Novel regulatory factors of MMP-13 expression in human osteoarthritic chondrocytes. Ann Rheum Dis 68: 976-982, 2009.

32. Li J and Buchner J: Structure, function and regulation of the hsp90 machinery. Biomed J 36: 106-117, 2013.

33. Whitesell L and Lin NU: HSP90 as a platform for the assembly of more effective cancer chemotherapy. Biochim Biophys Acta 1823: 756-766, 2012 